\title{
EXPERIENCIA SOBRE EDUCACIÓN RURAL EN EL MUNICIPIO DE SAMACÁ, BOYACÁ. COLOMBIA
}

\author{
María Cecilia Rodríguez Ruedal \\ Marco Antonio Piñeros Piñeros ${ }^{2}$
}

\section{Resumen}

La experiencia, que a continuación se describe, es el resultado del trabajo con comunidades e instituciones educativas rurales que viene desarrollando el Grupo de Investigación Educación Rural de la Universidad Pedagógica y Tecnológica de Colombia desde 1999, en el municipio de Samacá, Boyacá. Esta se realiza en dos fases: la primera, recoge una visión holística de carácter sociocultural y pedagógico de las familias que habitan en las veredas de Guantoque, Páramo Centro y Gacal y sus respectivas instituciones educativas. La segunda fase, coherente con los resultados obtenidos, busca diseñar y ejecutar, a través de la investigación-proyección social, una propuesta alternativa pedagógica y de atención primaria en salud, que con- tribuya a mejorar las condiciones de los menores y sus familias en el sector. La metodología utilizada en este proceso es la investigación-acción participativa, donde se han involucrado, los maestros de las escuelas, los padres y las madres de familia, las instituciones y organizaciones del municipio, la administración municipal y la Secretaria de Educación Departamental.

Los resultados obtenidos se relacionan con los compromisos adquiridos por los diferentes sujetos participantes, pero además, con el conocimiento de la realidad cultural, pedagógica y física de los menores.

Palabras claves: educación rural, investigación-acción, comunidad.

1 Licenciada en Enfermeria de la Universidad Industrial de Santander; Mg. en Desarrollo Educativo y Social de la Universidad Pedagógica Nacional-CINDE, Bogotá; profesora titular de la Universidad Pedagógica y Tecnológica de Colombia.

2 Licenciado en Psicologia y Pedagogia de la Universidad Pedagógica Nacional; Mg. en Investigación Educativa y Análisis Curricular de la Universidad Pedagógica Nacional; especialista en Psicologia Educativa de la Universidad Católica de Colombia; Docente Asociado de la Universidad Pedagógica y Tecnológica de Colombia.

Recibido: 3 de novembre-2006 A Aprobado: 30 de noviembre-2006 


\section{Abstract}

The author describes the experience of working with rural communities and its educational institutions developed by the Rural Education Investigative Group of Universidad Pedagógica y Tecnológica de Colombia, since 1999 at the municipality of Samacá, Boyacá. This experience had two stages; the first one gathered the socio cultural and pedagogical characteristics, in a holistic vision, of the families living in Guantoque, Páramo Central y Gacal areas. The second stage of the experience is based on the results obtained and searches through the investigation-social projection, the design and carrying out of an alternative proposal in pedagogy and health primary attention, which contributes to improve the living conditions of children and their families. The methodology used is based in the investigation-action paradigm, and has being adopted by teachers, parents, municipal institutions and organizations, as well as the administration and secretary of education of the municipality.

Keywords: rural education, action-investigation, community.

\section{Descripción general del proyecto}

- Tombres de los proyectos: Diseño de una propuesta pedagógica 1 alternativa para la educación rural del municipio de Samacá. Proyecto de atención primaria en salud, escuelas de Guantoque, El Llanito, Tibaquirá y colegio de La Libertad del municipio de Samacá.

- Modalidad de extensión: programas y megaproyectos que articulan simultáneamente formación, investigación y extensión.

- Profesores y estudiantes que participan: Grupo de investigación y extensión en Educación Rural de la Universidad Pedagógica y Tecnológica de Colombia. El grupo que viene desarrollando este proyecto de investigación-proyección social se denomina Educación Rural y está actualmente reconocido por la UPTC y COLCIENCIAS.

- Características generales de los proyectos: teniendo en cuenta que estos proyectos corresponden a una segunda fase del proceso de intervención, esta última intenta, mediante la investigación-acción participativa con profesores de las escuelas rurales de Guantoque y Tibaquirá y el colegio La Libertad, generar e implementar una propuesta pedagógica alternativa en dichas instituciones, que favorezca el desarrollo cognitivo, productivo y afectivo de los niños y las niñas y sus familias en el sector.

Se parte del diagnóstico resultante de la primera fase de investigaciónintervención, que hace un análisis holístico de la cultura y dentro de 
esta, de los patrones que dificultan el proceso de desarrollo en los menores. En este sentido, se trabajan cuatro categorías de análisis: familia (niñez y mujer), economía, medio ambiente y educación y pedagogía. A partir de estos resultados, se establecen dos proyectos de investigación-acción: Diseño de una propuesta pedagógicả altemativa para la educación rural del municipio de Samacá y Proyecto de investigaciónintervención en atención primaria en salud.

- Actores y/o sectores sociales en donde interactúan los proyectos: estos proyectos se vienen desarrollando en las veredas de Guantoque, Gacal, Páramo Centro y Tíbaquirá del municipio de Samacá, con participación de la Universidad Pedagógica y Tecnológica de Colombia y la Alcaldía Municipal; están vinculados los profesores de cuatro instituciones educativas, 30 en total; los niños y las niñas estudiantes, aproximadamente 620 menores, y casi 800 hogares, los padres y las madres de familia y organizaciones e instituciones sociales del sector.

- Ubicación geográfica: las veredas de Guantoque, Gacal, Páramo Centro y Tibaquirá se ubican a unos 28 kilómetros de la ciudad de Tunja, por la vía del Puente de Boyacá.

- Fecha de iniciación, tiempo de duración: el proyecto en su primera fase inició en el mes de agosto de 1999, y culminó en agosto del 2002. La segunda fase comenzó en febrero del 2003 y se encuentra actualmente en proceso.

\section{Definición de problemáticas y pertinencia social}

Los proyectos son el resultado del análisis de las condiciones socioculturales del entomo de las veredas de Guantoque, Páramo Centro, Gacal y Tibaquirá del municipio de Samacá; se obtuvieron las siguientes conclusiones, en las cuales se puede definir tanto la problemática como la pertinencia social de los proyectos.

$\mathrm{Al}$ establecer la correspondencia entre los objetivos y los hallazgos de la investigación, se encuentra que los factores socioculturales inciden directamente en las condiciones de aprendizaje y en la permanencia en el sistema escolar, de los niños y las niñas de las escuelas Guantoque, El Llanito y los colegios San Francisco de Asís y La Libertad.

Se evidenció que la dinámica interna de la familia, determinada por las formas de relaciones, roles, autoridad, pautas de crianza y trabajo, es un elemento cultural en el que predominan estructuras patriarcales rígidas y se 
establecen relaciones conflictivas cotidianas que, por el hecho de constituirse en proceso de socialización primaria, determinan patrones de comportamiento y conductas perdurables con efectos negativos en el desarrollo cognitivo, afectivo y valoral de los niños y las niñas.

Respecto a las condiciones físicas de los niños y las niñas, relacionadas con los procesos de crecimiento y desarrollo, sobresale que los aspectos nutricionales son deficientes en gran escala, por el escaso consumo de micronutrientes y, en general, de los componentes esenciales de los alimentos que, en una dieta balanceada, les permita un adecuado desarrollo integral de acuerdo con su edad, con el fin de responder a las exigencias de la escuela, de la familia y a su propia evolución biológica.

Asimismo, las condiciones de niños trabajadores y niñas trabajadoras son aspectos que supeditan la permanencia o deserción en el sistema escolar. Los menores del sector estudiado son vinculados tempranamente a las labores agrícolas, pecuarias y domésticas, ya sea como forma de apoyo a la economía familiar o como asalariados, situación que desvincula las condiciones motivacionales para que tengan como prioridad los procesos escolares. En este mismo orden, se presenta la escasa importancia que los padres de familia le dan a la educación escolar como elemento fundamental para el mejoramiento de los futuros hombres y mujeres, con calidad de vida.

Las prácticas de los adultos en contra del medio ambiente, entre las que se destacan el uso indiscriminado de agroquímicos y la contaminación de fuentes hídricas, de suelos y aire, deterioran la salud de los menores y los hacen vulnerables en relación con su integridad física y psicológica, lo que condiciona su desarrollo cognitivo. En esta perspectiva, su futuro es desesperanzador, dada la circunstancia prevaleciente de proyección cultural, donde las prácticas agrícolas que inciden en el deterioro ambiental son reproducidas, y ponen en riesgo un desarrollo económico y social sostenibles, lo cual aumenta los niveles de pobreza.

El análisis y la interpretación de los hallazgos de la investigación conducen a reflexionar acerca de la alta incidencia que los factores socioculturales tienen sobre los procesos educativos formales de los niños y las niñas; es decir, pensar la pedagogía y la educación requiere indispensablemente atravesar las fronteras de la escuela y converger los significados del mundo y la cotidianidad del contexto en el cual se desarrolla el niño.

En los campos educativo y pedagógico, se encontraron situaciones como: 
- En los procesos metodológicos, predominan los esquemas de educación tradicional, formalizada por las instancias superiores que son aplicadas conforme a las exigencias propuestas. Sin embargo, existen algunos profesores que han venido criticando y replanteando el modelo, proponiendo formas innovadoras, que se pueden constituir en puntos de partida para la generación de alternativas metodológicas apropiadas para el sector rural.

- En relación con la enseñanza del español y la matemática, los y las docentes resaltan la diferencia en el aprendizaje de estas dos áreas, en tanto afirman, que la primera resulta de dificil enseñanza en la medida en que pesa más la cultura que los procesos de asimilación. Por su parte, la matemática forma parte de su mundo cotidiano y se hace más fácil en su comprensión; no obstante, este aspecto conduce más a un aprendizaje pragmático de la ciencia, que hacia la construcción de un pensamiento riguroso y lógico.

- En cuanto a los recursos didácticos, se aprecia de manera positiva la elaboración y uso de estos con elementos del medio. Este aspecto favorece los procesos de aprendizaje en el sentido de que los recursos utilizados son familiares, porque forman parte de la cultura; si se parte del hecho de que los recursos didácticos deben propiciar un acercamiento entre la palabra y la realidad, se constituye en un factor favorable en la construcción de conceptos y del aprendizaje mismo.

Para cada área, existe una gran y muy variada gama de recursos didácticos que van desde el texto guía, cuadernos y fichas, hasta actividades lúdicas; muchas de ellas son elaboradas por el o la docente y sus estudiantes, lo cual muestra sus habilidades recursivas, para superar las limitaciones de material didáctico de la escuela.

Se concibe la motivación como el proceso que estimula al estudiante y lo compromete con el aprendizaje escolar; en este sentido, se encuentra que hay preocupación por parte de los y las docentes por lograr una efectiva participación durante todo el proceso del aprendizaje; este se caracteriza por el método de enseñanza que se da en la apertura, proceso y cierre de la clase, donde la comunicación queda establecida en el acto motivacional por parte del profesor, quien establece las reglas de juego en cada una de las etapas de la clase. En este orden, la motivación 
es exclusiva del docente y depende, en cierta forma, de su personalidad y sus habilidades pedagógicas.

- Según la concepción del modelo pedagógico, se tiene que los maestros en la zona identifican este con las metodologías, la estructura de la institución y el enfoque del conocimiento, centrándolo en teorías de corte tradicional, ecléctico y constructivista. Señalan como elementos constitutivos del modelo, el diagnóstico como punto de partida, los objetivos entendidos como los logros por alcanzar en cuanto a autoestima, respeto, reflexión, paz y liderazgo; metodología de enseñanza y estrategias para llevar a cabo la enseñanza y el aprendizaje. Esto implica una concepción fundada en valores específicos, ligada, de alguna manera, a los actuales problemas sociales, económicos y políticos del país.

- La evaluación se hace para definir la disposición que tienen los niños y las niñas en cada uno de los saberes y para analizar, de manera crítica, los procesos de enseñanza, aunque, también para establecer el grado de memorización y mecanización de la información, abriendo la posibilidad de una evaluación orientada a los distintos aspectos del desarrollo no solo cognitivo, sino afectivo, de comportamiento y actitudinal.

- Se observa que un gran número de niños y niñas, con graves dificultades de aprendizaje, asisten a las instituciones estudiadas; estas son consecuencia de situaciones intrasíquicas, nutricionales, culturales, económicas y pedagógicas, situación que se hace difícil en la medida que los y las docentes carecen de formación especializada para atenderlas.

\section{Apuesta ética y social}

El grupo de investigación y extensión Educación Rural plantea como misión: Mediante los procesos investigativos y de trabajo comunitario que se deriva de este, se reconocen las realidades locales y culturales, y favorece el fortalecimiento del desarrollo social y educativo por medio de proyectos innovadores para el área rural que mejoren la calidad de vida de la población.

La visión del grupo retoma para su carácter, la establecida por la Universidad Pedagógica y Tecnológica de Colombia. Diseñará y ejecutará proyectos de investigación y extensión que fortalezcan los procesos locales en el área del desarrollo comunitario y pedagogía de lo rural.

Desde esta perspectiva, se alimenta de la misión y la visión de la Universidad Pedagógica y Tecnológica de Colombia, y desde allí es coherente con las propuestas de los diferentes programas académicos que le sirven 
de soporte para su desarrollo: los programas de Psicopedagogía, Medicina, Enfermería, Medicina Veterinaria, Ingeniería Agronómica y la Maestría en Desarrollo Rural.

\section{Antecedentes y objetivos}

Los proyectos en mención parten de una primera fase diagnóstica de carácter sociocultural y educativo, cuyos resultados orientan una segunda fase, que se hace posible a través de los proyectos descritos.

Desde la perspectiva académica, la construcción de procesos educativos innovadores y de propuestas en atención primaria en salud, buscan transformar la realidad, pero además, generar un discurso científico que establezca nuevas posibilidades de desarrollo para el mejoramiento de la calidad de vida de las poblaciones. De igual manera, a partir de este, se pretende establecer un soporte para el aprendizaje desde la experiencia, coherente con las realidades, los problemas y necesidades de los ámbitos local, regional y nacional. Es una adecuada forma de construir conocimiento desde la academia.

El proyecto Diseño de una propuesta pedagógica alternativa para la educación rural de Samacá plantea el siguiente objetivo general: Diseñar una propuesta pedagógica alternativa con la comunidad educativa que articule los procesos académicos, productivos, sociales, culturales, ambientales y de salud, con el propósito de conseguir un desarrollo rural sostenible de los niños, las niñas y las familias del municipio de Samacá (Boyacá).

Los siguientes son los objetivos específicos:

- Formar a los maestros y otros miembros de la comunidad educativa como investigadores y gestores para el diseño de la propuesta pedagógica alternativa para la educación rural.

- Establecer conjuntamente con la comunidad educativa, en especial con los profesores, los elementos conceptuales y teóricos que sirven de derrotero para la generación de la propuesta pedagógica alternativa.

- Crear un banco de datos con las iniciativas de las comunidades educativa y social que favorezca la construcción común del proyecto educativo, productivo y de salud como marco de referencia y soporte de la propuesta pedagógica.

- Establecer conjuntamente con la comunidad educativa, los criterios pedagógicos, didácticos y metodológicos que articulen los procesos académicos, productivos, sociales, culturales, ambientales y de salud, 
que permitan, desde la escuela, la generación de nuevos procesos educativos y sociales.

- Sistematizar la experiencia teniendo como eje central el proceso y dinámica del trabajo del grupo gestor y la formulación de la propuesta pedagógica alternativa para la educación rural.

Coherente con este proyecto, se diseña el Proyecto de investigación en atención primaria en salud para las escuelas de Guantoque, El Llanito, Tibaquirá y el colegio de La Libertad del municipio de Samacá, que nos permite afrontar los problemas del desarrollo del niño desde los ámbitos pedagógicos y del desarrollo cognitivo, pero además, es la comprensión de las relaciones intersubjetivas y culturales ligadas a estos procesos. Los dos proyectos están estrechamente interrelacionados.

Se establece para este proyecto, el siguiente objetivo general: Implementar las estrategias de escuelas saludables: empoderamiento comunitario, desarrollo de actitudes personales y creación de entornos saludables, a través de procesos de educación, promoción de la salud y el medio ambiente en la búsqueda de desarrollo humano integral de los y las escolares, sus familias y la comunidad.

En función de este proceso, evaluar el impacto logrado hasta el año 2005, y sistematizar la experiencia, en las instituciones educativas del Colegio La Libertad y las escuelas de El Llanito, Guantoque y Tibaquirá de las veredas del Gacal, Páramo Centro y Guantoque del municipio de Samacá.

Los siguientes son los objetivos específicos:

- Valorar y correlacionar las condiciones nutricionales, de desarrollo y crecimiento y cognitivo, con los procesos de aprendizaje de los niños y las niñas desde 0 a los 12 años y en función de esto, generar estrategias saludables con miras a mejorar sus condiciones de vida.

- Promover la participación de la comunidad mediante procesos educativos y de sensibilización, encaminados al empoderamiento y concientización en la generación de estilos de vida saludables para los niños y las niñas de las instituciones educativas y sus familias.

- Fomentar actitudes personales mediante procesos educativos y de sensibilización a padres de familia, profesores y estudiantes acerca de la importancia nutricional, el saneamiento del entorno ambiental, social y familiar para el desarrollo integral de los niños y las niñas. 
- Evaluar los logros y dificultades del proceso, en la búsqueda del impacto obtenido mediante la implementación de las estrategias.

- Sistematizar las experiencias de trabajo comunitario en salud, teniendo como objeto del proceso las metodologías utilizadas para la intervención, impacto y resultados obtenidos.

\section{Estrategia metodológica}

Los proyectos se sustentan en la teoría crítica, la educación popular, entre las que se reconocen las propuestas de Paulo Freire, la Teoría de la Acción Comunicativa de Jürgen Habermas y las pedagogías activas.

\section{Estrategia pedagógica}

La ejecución de los proyectos es posible mediante la vinculación de estudiantes al proceso. En ese sentido, en el transcurso de los cinco años de trabajo en el sector, se han desarrollado los siguientes proyectos de grado con estudiantes de los programas de Psicopedagogía y Enfermería, especialización en Psicología Educativa y trabajo de año sabático:

- Las teorías implícitas, de los maestros de educación primaria básica rural, acerca de la enseñanza. Respaldado por: Marco Antonio Piñeros Piñeros. Especialización en Psicología Educativa.

- Estrategias pedagógicas para promover la lectoescritura en los niños de 2 a 5 años, Escuela El Llanito, municipio de Samacá. Escuela de Psicopedagogía, noviembre del 2001. Respaldado por: Mariana Pulido Rodríguez, Martha Cecilia Rivera López y Nelsy Soraya Gómez Pérez. Psicopedagogía.

- Propuesta pedagógica Alternativa para la Educación Rural. Junio del 2002. Respaldado por: Yenny Patricia Salamanca Sánchez, Yudy Esperanza Forero Gómez y Astrid Ofelia Buitrago Vivas. Psicopedagogía.

- La escuela taller didáctico para la comunidad educativa. Escuela Nueva Guantoque, Samacá. Respaldado por: Ruth Adriana Córdoba y Ana Cecilia Gómez.

Del programa de Enfermería, se han desarrollado los siguientes proyectos relacionados con el desarrollo académico de los participantes: 
- Condiciones de salud y medio ambiente según el uso de agroquímicos de la vereda de Páramo Centro del municipio de Samacá. Respaldado por: Miguel Ángel Vega Álvarez. Escuela de Enfermería.

- Algunos imaginarios en salud de una comunidad rural del municipio de Samacá. Respaldado por: María Cecilia Rodríguez Rueda.

En el área de salud, se han desarrollado prácticas de salud comunitaria, valoración de crecimiento y desarrollo y es campo de práctica en la rotación para médicos internos en el área de Atención Primaria en Salud. De igual manera, los y las estudiantes de enfermería han tenido la oportunidad de desarrollar algunas actividades relacionadas con este campo.

Desde esta perspectiva, la práctica y experiencia de campo en este sector se constituyen en un importante recurso académico, investigativo y de proyección social, que dan cabida a la discusión y desarrollos conceptuales que permiten construir nuevas miradas críticas en función de la transformación social. Por ello, el campo de acción de Samacá es un laboratorio social, donde se tejen diversas y nuevas realidades, en medio del constante diálogo de saberes.

\section{Elementos relevantes del proceso}

Considerando que los proyectos forman parte de dos líneas de investigación que el grupo Educación Rural viene desarrollando, estos tienen una orientación multi e interdisciplinaria. Se han establecido contactos con diferentes instancias que desarrollan propuestas en el sector rural. Son proyectos eminentemente interdisciplinarios, en la medida que convergen en ellos, diversos programas de la Universidad, entre otros: Psicopedagogía, Medicina, Enfermería, Ingeniería Agronómica, Medicina Veterinaria y la Maestría en Desarrollo Rural. De igual manera, en el interior de la Universidad existen algunos grupos con líneas de investigación, cuyos perfiles se encuentran con la propuesta del grupo Educación Rural: Grupo Asociado para el Desarrollo - GIPA y KALLAWAYA y BACHUE.

Las propuestas son interinstitucionales, entre organismos e instituciones sociales y ONGs del municipio, juegan un papel muy importante en la generación participativa de propuestas de intervención y mejoramiento. 


\section{Fuentes y usos de recursos}

La sostenibilidad de los proyectos es posible gracias al capital semilla que la Universidad Pedagógica y Tecnológica de Colombia aporta, y en principio, al convenio UPTC-COLCIENCIAS; actualmente se está ejecutando en convenio con el municipio de Samacá.

\section{Resultados obtenidos y esperados}

En la primera fase, se realizó un diagnóstico de los factores socioculturales y pedagógico-educativos de las veredas de Guantoque, Páramo Centro y Gacal del municipio de Samacá y sus respectivas instituciones educativas. Los resultados de este trabajo se encuentran en el libro SENTIDO Y CULTURA: Realidad de la Escuela Rural, tres artículos publicados en las revistas Pensamiento y Acción, Apuntes de CENES y Revista de Enfermería de la Universidad Nacional. Se participó con ponencias nacionales y una ponencia internacional en la ciudad de Santiago de Chile en abril del 2003.

En la segunda fase de los proyectos, se ha logrado comprometer a la administración municipal y a la Secretaría de Educación Departamental, aspecto que facilita el trabajo con los maestros de las instituciones educativas comprometidas. De igual manera, las actividades con los y las docentes de las instituciones se fundamentan en la construcción de microproyectos de aula, orientados a consolidar una propuesta general, a partir de la implementación de pequeñas experiencias, que respondan al desarrollo cognitivo de niños y niñas; además, contribuyen desde allí con la solución de problemas del entomo, relaciones intra y extrafamiliares, condiciones ambientales, productividad y economía, entre otros.

Es importante resaltar, como la dinámica del proceso en la ejecución de los proyectos ha facilitado la consolidación del grupo Educación Rural de la Universidad Pedagógica y Tecnológica de Colombia, al cual se han vinculado 30 maestros rurales y es reconocido actualmente por las comunidades, instituciones, ONGs del sector y el municipio. 


\section{REFERENCIAS}

Universidad Pedagógica y Tecnológica de Colombia. (2003). Sentido y Cultura: Realidad de la Escuela Rural. Colombia: UPT.

Universidad Pedagógica y Tecnológica de Colombia. Revistas Pensamiento y Acción. Colombia: UPT.

Universidad Nacional. (2004). Revista de Enfermería. Colombia. 